\title{
BACLOFEN INDUCED SUICIDALITY IN A PATIENT WITH ALCOHOL DEPENDENCE SYNDROME: A CASE REPORT WITH REVIEW OF LITERATURE
}

\author{
Shree Mishra ${ }^{1}$, Santanu Nath ${ }^{1,2}$ \& Susanta Kumar Padhy ${ }^{1}$ \\ ${ }^{1}$ Department of Psychiatry, All India Institute of Medical Sciences (AIIMS), Bhubaneswar, Odisha, India \\ ${ }^{2}$ All India Institute of Medical Sciences, Deoghar, Jharkhand, India
}

received: 15.10.2020;

revised: 8.1.2021;

accepted: 12.2 .2021

\section{INTRODUCTION}

Baclofen is a GABA-B agonist, used mainly in the treatment of spasticity due to its myorelaxant properties. Preclinical and clinical studies have demonstrated the efficacy of baclofen in the treatment of alcohol use disorders (AUD). It is well tolerated having certain adverse effects of fatigue, somnolence, tinnitus, paresthaesias and blurred vision (Beaurepaire et al. 2019). There are reports of baclofen overdosing resulting in encephalopathy, respiratory depression, muscular hypotonia, coma and delirium (Holla et al. 2015). Reports of psychiatric side effects are rare and till date there are paucity of reports of suicidality occurring in the context of treatment with baclofen. We report a case of alcohol dependence syndrome who was treated with baclofen as an anticraving agent and who developed suicidal ideations with it.

\section{CASE PRESENTATION}

A 36-year-old married male, hailing from a lower socioeconomic background, with no significant past, personal and family history was brought with complaints of inability to recognize family members, irrelevant talks, fearfulness along with tremulousness of his hands for last 1 day. History revealed that he had stopped drinking alcohol for last 2 days which he had been drinking regularly for last 10 years in a dependence pattern. On examination, he was found to be have normal vital signs with disorientation to time, place and person, fluctuating attention and concentration, increased psychomotor activity, hallucinosis and tremors of the whole body most prominently in both extended hands. A provisional diagnosis of alcohol dependence syndrome, currently in withdrawal state with delirium tremens (DT) (F10.41) (ICD-10) (World Health Organization 1992) was made and he was admitted. On admission, relevant blood investigations and CT Scan brain was done which were unremarkable and he was started on oral Chlordiazepoxide in a symptom-triggered regimen for targeting withdrawal along with parenteral Thiamine. His delirium and other withdrawal symptoms gradually waned off completely within next few days and he was started on tablet Baclofen $20 \mathrm{mg} / \mathrm{day}$ as an anticraving henceforth. Two days after starting Baclofen, he started to express suicidal ideations (Beck Scale for Suicide Ideation score (BSI) 26) (Beck et al. 1979) which was occurring to him three to four times/ day and was associated with plans to execute it, although this was not associated with any depressive cognitions or any other mood or perceptual disturbances cross-sectionally. Baclofen was temporarily stopped thinking of a possible link between the two and the patient was kept under close supervision. A day after discontinuing Baclofen, his suicidality substantially decreased as evidenced by a decrement of BSI score to 5 in next 3 days. Days after, a detailed evaluation was planned and this revealed long standing symptoms suggestive of depression with occasional death wishes that were triggered by his financial loss and family interpersonal issues. This mood state was reportedly also present when he would remain completely abstinent from alcohol for at least a month. There was no suicidality in the preceding 3 months before this index presentation and they escalated abruptly on starting baclofen and reduced after stopping the same. A plan to restart Baclofen was aborted considering ethical issues in retriggering another suicidal ideation. We applied the Naranjo Adverse Drug Reaction Probability Scale (Naranjo et al. 1981) that yielded a score of 6, suggesting 'probable' causality due to Baclofen. He was discharged with tablet Escitalopram $10 \mathrm{mg} /$ day for his depressive symptoms along with Acamprosate as an anticraving and oral Thiamine and a follow up visit after a month revealed that he was maintaining abstinence with improved mood state and no return of suicidality. During this time he was also on psychosocial interventions from our team of social workers. He was subsequently planned for a relapse prevention therapy.

\section{DISCUSSION}

The index case developed suicidality 2 days after starting Baclofen which quickly remitted upon discontinuing the same. He was not delirious during this time which negates the possibility of DT triggering a suicidal 
Table 1: Psychiatric adverse effects of baclofen when used in AUD

Psychiatric adverse effects of baclofen use in alcohol use disorders

\author{
Confusion (Auffret et al. 2017) \\ Sedation (Auffret et al. 2017) \\ Seizures (Auffret et al. 2017) \\ Coma (Auffret et al. 2017) \\ Agitation (Auffret et al. 2017) \\ Behavioural disorders (Auffret et al. 2017) \\ Hallucinations (Auffret et al. 2017)
}

Space-time disorientation (Auffret et al. 2017)

Baclofen withdrawal syndrome (Auffret et al. 2017)

Mania (Geoffroy et al. 2014)

Psychosis (Leandre \& Ginory 2018)

Catatonia (Thippeswamy et al. 2017)

Suicidal behaviour (when comorbid psychiatric disorder present) (Beaurepaire et al. 2019)

behaviour in the patient. He however had a history of chronic depression before the current presentation but in the recent past he was not expressing death wishes until 2 days after starting Baclofen. We thus propose that it was Baclofen which possibly triggered the suicidal ideation.

There have been reports of mania (Geoffroy et al. 2014), psychosis and catatonia occurring in the context of baclofen withdrawal (Leandre \& Ginory 2018) and overdose (Thippeswamy et al. 2017). Caution has been exercised about the risk of Baclofen intoxication in many reports till date but none so far have reported baclofen to be inducing a suicidal behaviour as in our patient. In 2012, the French Drug Agency issued a pharmacovigilance report about a possible risk of increased suicidal behaviours among AUD subjects on Baclofen though this was never confirmed (Villier et al. 2012). It is known that AUD patients have psychiatric comorbidities that can trigger suicidal behaviours, thus skewing the unadjusted findings that Baclofen treatment is associated with an increased risk of suicidal behaviors. Beaurepaire et al (2019) in a recent systematic review also exercised caution about the use of Baclofen stating that it can induce suicidality when comorbid psychiatric illness is present. He also cautioned against the use of baclofen in those having a history of such behaviour (Beaurepaire et al. 2019). Such a possibility cannot be ruled out in our case since our patient had comorbid depressive disorder which was independent of his alcohol intake pattern.

A search for the neurobiological causation of suicidality by Baclofen remains distant since nothing can be proposed conclusively. GABA-ergic neurotransmitter system has been implicated for both depression and suicidal behaviour though such an opinion is conflicting (Carballo et al. 2008). However, a prominent role of the glutamatergic and GABA-ergic neurotransmitter systems and the deficiency of Glutamine Synthetase enzyme in immunohistochemical study of key brain areas in suicidal behaviour has been proposed by Bernstein et al. (2013). Taking into consideration both the above, we propose that, on a background of chronic depression, baclofen, a GABA-B agonist could have possibly triggered the already perturbed neurotransmitter imbalance due to the recent alcohol withdrawal state (a glutamatergic hyperexcitable state) in our patient culminating into suicidal behaviour. Baclofen has also been found to alter dopaminergic neurotransmission. GABA-B receptors, located in ventral tegmental area, are present both on the cell body of dopamine neurons and the terminals of glutamatergic afferent neurons. Their activation by GABA-B receptor agonist may exert an inhibitory action on dopamine neurons (Yoshida et al. 1994). Baclofen thus has been implicated in decreasing dopamine release and transmission. There are conflicting evidences of dopaminergic neurotransmission in suicidal behaviour being mediated through aggression, link can be drawn here between hypo-dopaminergic state induced by Baclofen and the dopaminergic alterations in suicidal behaviour which we propose could have summated to the suicidal behaviour that our case showed. Whatever the mechanism might be, this case highlights the need for psychiatrists to exert caution while prescribing Baclofen as an anti-craving agent, especially in patients with co-morbid major psychiatric disorders and past history of suicidal attempts. Future studies are warranted that will focus more on this novel anticraving agent in terms of its mechanism of action and its neurobehavioural side effects.

\section{Acknowledgements: None.}

\section{Conflict of interest: None to declare.}

\section{Contribution of individual authors:} patient under question and treated him, wrote the introduction and the discussion portion of the manuscript.

Santanu Nath \& Susanta Kumar Padhy the case description of the manuscript was written, the final check and correction was done and was acknowledged by all the authors before submission.

\section{References}

1. Auffret M, Labreuche J, Duhamel A, Deheul S, Cottencin $O$, Bordet $R$, et al. Proactive regional pharmacovigilance system versus national spontaneous reporting for collecting safety data on concerning off label prescribing practices: an example with baclofen and alcohol dependence in france. Drug Saf 2017; 40:257-62 impulsivity and neuroticism (Carballo et al. 2008). A

Shree Mishra \& Santanu Nath took consent from the 
2. Beaurepaire RD, Sinclair JMA, Heydtmann M, Addolorato G, Aubin H-J, Beraha EM, et al. The Use of Baclofen as a Treatment for Alcohol Use Disorder: A Clinical Practice Perspective. Frontiers in Psychiatry 2019; 9

3. Beck AT, Kovasac M, Weissman A. Assessment of suicidal intention: the Scale for Suicide Ideation. J Con- sult Clin Psychol 1979; 47 (2): 343-52.

4. Bernstein HG, Tausch A, Wagner R, Steiner J, Seeleke P, Walter $M$, et al. Disruption of glutamate-glutamine-GABA cycle significantly impacts on suicidal behaviour: survey of the literature and own findings on glutamine synthetase. CNS Neurol Disord Drug Targets 2013; 12:900-13

5. Carballo JJ, Akamnonu CP, Oquendo MA. Neurobiology of Suicidal Behavior. An Integration of Biological and Clinical Findings. Archives of Suicide Research 2008;12:93-110.

6. Geoffroy PA, Auffret M, Deheul S, Bordet R, Cottencin O, Rolland B: Baclofen-induced manic symptoms: Case report and systematic review. Psychosomatics 2014; 55:326-32

7. Holla B, Gowda GS, Prabhu L, Baby S, Viswanath B, Chand $P$, et al. High doses of Baclofen as suicide attempt in patients with alcohol use disorders - A serious concern. Asian Journal of Psychiatry 2015; 17:99-100
8. Leandre F, Ginory A. A case of Baclofen withdrawal induced psychosis treated with lorazepam. Australian \& New Zealand Journal of Psychiatry 2018; 53:174

9. Naranjo CA, Busto U, Sellers EM, Sandor P, Ruiz I, Roberts EA, et al.: A method for estimating the probability of adverse drug reactions. Clinical Pharmacology and Therapeutics 1981; 30:239-45

10. The ICD-10 Classification of Mental And Behavioural Disorders. 1st ed. Geneva: World Health Organization, 1992. Print

11. Thippeswamy H, Nahar A, Reddy MS, Subramaniyam B, Chandra P, Chaturvedi S. Baclofen overdose presenting as psychosis with catatonia. Indian Journal of Psychological Medicine 2017; 39:695-697

12. Villier C, Schir E, Mallaret M. Adverse Effects of Baclofen in the Treatment of Addictions: 2011 National Pharmacovigilance Monitoring 2012. Available online at: https://ansm.sante.fr/var/ansm_site/storage/original/appli cation/264282cafe82f9elf08323df4722fdal.pdf

13. Yoshida M, Yokoo H, Tanaka T, Emoto H, Tanaka M. Opposite changes in the mesolimbic dopamine metabolism in the nerve terminal and cell body sites induced by locally infused baclofen in the rat. Brain Res 1994;636:111 44

Correspondence:

Assistant Professor Santanu Nath, MBBS; MD; DNB

Department of Psychiatry, All India Institute of Medical Sciences (AIIMS)

Bhubaneswar, Sijua, Patrapada, P.O: Dumduma, Odisha, 751019, India

E-mail: beta.santanu@gmail.com 